\title{
Comparative Clinical Effectiveness Research Focused on Community-Based Delivery of Palliative Care: Overview of the Patient-Centered Outcomes Research Institute's Funding Initiative
}

\author{
Carly P. Khan, PhD, MPH, RN, Stephanie Parver, MPH, CPHQ, Julie Kennedy Lesch, MPA, \\ Kimberly DiGioia, MS, Bridget Gaglio, PhD, MPH, Sarah Daugherty, PhD, MPH, \\ Steven B. Clauser, PhD, MPA, and Neeraj K. Arora, PhD
}

\begin{abstract}
Palliative care is a growing specialty that addresses the needs of individuals diagnosed with advanced illness and their caregivers. Although palliative care has been shown to improve a variety of patient- and caregivercentered outcomes, access to comprehensive palliative care services for patients is often limited. There is a need to identify the most effective approaches to delivering palliative care to patients in community settings. In fiscal year 2017, based on extensive input from a diverse set of stakeholders, the Patient-Centered Outcomes Research Institute (PCORI) funded nine multisite comparative clinical effectiveness research (CER) trials focused on community-based delivery of palliative care for a total investment of $\$ 80$ million. These studies, focusing on advance care planning and models of palliative care delivery, represent some of the largest most complex palliative care trials funded to date. Each study evaluates both patient and caregiver outcomes, and together, these trials include a broad range of health conditions, interventions, and settings of care. PCORI has also fostered a learning network of the funded awardees to facilitate the successful conduct of these CER studies and to support awardee efforts to develop collaborative products relevant to advancing the field of palliative care research and practice. The protocols of each of the nine trials, detailed in this issue, demonstrate the expansive reach of the investment PCORI has made in an effort to further the research agenda and provide substantive research evidence in stakeholder-identified areas of need in the field of palliative care.
\end{abstract}

Keywords: advance care planning; advanced illness research; models of palliative care delivery; outcomes research; palliative care; Patient-Centered Outcomes Research Institute (PCORI)

\section{Background}

A DUlTs With ADVANCED ILLNESSES, as well as their caregivers, experience a high burden of physical and psychosocial symptoms associated with their diagnosis and treatment. ${ }^{1}$ Palliative care consists of several core components, including systematic assessment and management of patient symptoms, psychosocial support for patients and caregivers, advance care planning, and facilitation of goalconcordant care through coordinated services provided by clinicians. ${ }^{1,2}$ Patients who receive palliative care services experience significant improvements in their quality of life, fewer hospitalizations and emergency department visits, and in some cases, improved survival; palliative care has also been shown to result in reduced caregiver burden and improved caregiver quality of life. ${ }^{3-6}$ Clinical practice guidelines also call for early integration of palliative care to optimize outcomes among patients and caregivers. ${ }^{2}$

The field of palliative care, although relatively new, has become one of the fastest growing medical specialties in recent years. A significant proportion of hospitals in the United States today staff palliative care teams and evidence supports that delivery of palliative care both improves quality and decreases costs. ${ }^{7}$ However, access for patients to comprehensive palliative care services is often limited to institutional settings such as inpatient hospitals or hospices. The 
focus of palliative care delivery has predominantly been on patients who are very sick and have been hospitalized or are at the end-of-life stage of their illness. There is an unmet need for improving access to these services in community settings so that patients and their caregivers are supported when they are living with advanced illnesses and not necessarily dying from them. ${ }^{7}$ Furthermore, the proportion of older adults in the population continues to grow, which places increased urgency on the necessity for innovation in care delivery to meet the increased projected need for palliative care services in the future. ${ }^{8}$

Multiple systematic reviews, research, and policy reports acknowledge that although the efficacy of several palliative care-related interventions has been established, there is a need for systematic head-to-head comparisons of these interventions with a focus on more patient- and family-centered outcomes. Specifically, these reports call for comparative clinical effectiveness research (CER) on evaluating different models of palliative care delivery involving multiple care settings, providers of care, modes of care delivery, including populations other than those living with cancer, and longer follow-up periods. ${ }^{9,10}$ In addition, there is a need for CER evaluating different approaches to advance care planning that go beyond completion of advance directives and focus on the longitudinal care planning process. These should include evaluations that identify effective elements of advance care planning that promotes goal-concordant care and can be integrated into the workflow of standard care for patients with advanced illnesses. ${ }^{1,11}$ Furthermore, the National Institute of Nursing Research has called for future palliative care research to be conducted with systematic and significant stakeholder engagement to achieve impactful changes to care delivery for patients with advanced illnesses and their caregivers. ${ }^{12}$ Finally, there is a need to ensure equitable availability of palliative care for vulnerable populations, including rural and other groups experiencing disparities in access to palliative care. ${ }^{2}$

The Patient-Centered Outcomes Research Institute (PCORI) is a nonprofit independent nongovernmental organization charged with producing useful relevant clinical evidence through the funding of CER with a strong patientcentered focus. ${ }^{13,14}$ PCORI is committed to transparency and a rigorous stakeholder-driven process that emphasizes stakeholder engagement using a variety of forums and public comment periods to obtain public input to enhance its work. Given PCORI's focus on funding patient-centered CER studies that require sustained stakeholder input and engagement, several organizations, such as the National Academy of Medicine and the Center to Advance Palliative Care, have identified PCORI as an important source of funding for the next generation of palliative care research. ${ }^{1,7}$

In response to these recommendations and unmet research needs, PCORI identified CER on palliative care as a priority area for funding. ${ }^{15}$ In March 2016, PCORI convened a large multistakeholder palliative care workgroup meeting attended by 82 stakeholders that included patients, patient and caregiver advocates, clinicians, palliative care researchers, representatives of public and private payers, federal and private research funding organizations, clinical specialty societies, and industry representatives. The public was also invited to listen to the discussion at the workshop. Recommendations from this workgroup resulted in the development of a tar- geted PCORI-funding announcement (TPFA) that called for CER proposals focused on facilitating the delivery of community-based palliative care. ${ }^{16,17}$ In fiscal year 2017 (FY2017), PCORI funded seven CER trials as part of this TPFA; three trials focused on advance care planning and four focused on community-based models of palliative care delivery. In addition, in FY2017, PCORI also funded two investigator-initiated CER trials that focused on evaluating different models of delivering palliative care outside of hospital settings. Thus, in FY2017 alone, PCORI invested $>\$ 80$ million in nine large multisite palliative care CER trials. Collectively, these nine trials are some of the largest most complex community-based CER trials in the field of palliative care to date. Protocols from these nine studies are featured in this issue of the Journal of Palliative Medicine.

\section{Characteristics of the Funded Studies}

This set of studies will inform several unmet needs in the field of palliative care research. They will have a wide reach in terms of populations and settings, as well as a diverse set of interventions and delivery modalities being compared, and extensive engagement of a multitude of stakeholders. Three of the nine studies have a specific focus on advance care planning for patients with serious illnesses, and six studies focus on evaluating different models for delivering palliative care to patients and their caregivers in a variety of care settings (see Table 1 for research questions addressed in each study).

These studies include patients with advanced illnesses that require further palliative care research evidence. ${ }^{9}$ This encompasses those with cancer, end-stage renal disease, endstage liver disease, chronic obstructive pulmonary disease, heart failure, and neurodegenerative diseases, among others. In total, $>18,000$ patients will take part in these studies across 180 clinical sites in 27 U.S. states and Canada. For six of the nine studies, both English- and Spanish-speaking individuals were eligible to participate. The settings for recruitment and delivery of the interventions include primary care clinics, hospitals and emergency departments, outpatient specialty offices, and in-person and virtual visits to the patients in their homes. Some of the shared patient-centered outcome measures across the studies include health-related quality of life, symptom burden, depression, and anxiety. Other outcomes being collected focus on health care utilization, goal concordance of care, satisfaction with care, and decisional conflict. The larger sample sizes of the funded studies will allow for evaluation of heterogeneity of treatment effects (HTEs), to assess whether some patients have better outcomes than others. Some of the planned HTE analyses for these studies include subgroups by type of advanced illness, sociodemographic characteristics (age, gender, race/ethnicity, and language spoken), as well as multiple chronic conditions or number of comorbidities. Given the recommendation for longer follow-up periods in future research, most of these studies follow patients and their caregivers for at least 12 months and up to 24 months.

Another important characteristic of PCORI's palliative care funding announcement, based on strong recommendations from stakeholders, was the requirement to enroll caregivers along with patients and to evaluate the impact of interventions on caregiver outcomes. As palliative care 
Table 1. Research Questions

\begin{tabular}{lc}
\hline Study focus & Principal investigator(s) \\
\hline $\begin{array}{l}\text { Community-based } \\
\text { models of } \\
\text { palliative care }\end{array}$ & $\begin{array}{c}\text { Jennifer S. Temel, MD \& } \\
\text { Joseph A. Greer, PhD, MS, BS }\end{array}$ \\
& \\
& Huong Q. Nguyen, PhD, RN \& \\
& Richard A. Mularski, MD, MSHS, MCR
\end{tabular}

Richard A. Mularski, MD, MSHS, MCR

Victor Navarro, MD \& Manisha Verma, MD, MPH

Rebecca A. Aslakson, MD, PhD, FAAHPM, FCCM

Susan Enguídanos, PhD, MPH

Corita R. Grudzen, MD, MSHS, FACEP

Advance care planning
Neil S. Wenger, MD, MPH \& Anne M. Walling, MD, PhD
What is the comparative effectiveness of early integration of palliative care delivered by palliative care specialists through telemedicine vs. in-person clinic visits on patient- and caregiver-centered outcomes among patients newly diagnosed with advanced lung cancer and their caregivers?

What is the comparative effectiveness of a standard HBPC model that includes routine home visits made by nurses and palliative care providers vs. a tech-supported HBPC model that promotes early remote access to a provider during the nurse's visit to the patient's home on patientand caregiver-centered outcomes among older adults with advanced illnesses and their caregivers?

What is the comparative effectiveness of palliative care delivered by board-certified palliative care specialists vs. hepatologists trained in palliative care on patientand caregiver-centered outcomes among patients with end-stage liver disease and their caregivers?

What is the comparative effectiveness of surgeonpalliative care team comanagement vs. surgeon management alone on patient- and caregiver-centered outcomes among patients undergoing major cancer surgery and their caregivers?

What is the comparative effectiveness of home-based delivery of palliative care vs. palliative care provided by trained primary care clinicians in clinic on patientand caregiver-centered outcomes among patients with advanced illnesses and their caregivers?

What is the comparative effectiveness of palliative care delivered by a nurse-led telephonic case-management model vs. in person, outpatient specialty palliative care on patient- and caregiver-centered outcomes among patients with advanced illnesses who are discharged to home after an emergency department visit and their caregivers?

What is the comparative effectiveness of an AD alone vs. the AD plus a website designed to increase patients' readiness to engage in advance care planning (PREPARE website) vs. the AD plus the PREPARE website plus a care coordinator intervention on patientand caregiver-centered outcomes among primary care adult patients living with advanced illnesses and their caregivers?

Annette M. Totten, PhD, MPA \& France Légaré, B. Sc. Arch, MD, $\mathrm{MSc}, \mathrm{PhD}$, CCFP, FCFP

Kimberly S. Johnson, MD
What is the comparative effectiveness of a team-based vs. singular clinician approach to advance care planning using the SICP in the primary care setting among patients with advanced illnesses and their caregivers?

What is the comparative effectiveness of a structured ACP approach (Respecting Choices) vs. a patientguided self-management approach (Five Wishes) in facilitating formal or informal ACP within racial subgroups of patients and in reducing racial disparities between these groups on patient- and caregivercentered outcomes among older adult patients with advanced illnesses and their caregivers?

\footnotetext{
${ }^{a}$ Research questions are listed in order of corresponding articles included in this journal issue.

ACP, advance care planning; AD, advance directive; HBPC, home-based palliative care; SICP, Serious Illness Care Program.
}

moves beyond the inpatient hospital setting to outpatient community settings, supporting caregivers will be an important aspect of quality care. ${ }^{17,18}$ Consequently, across these nine CER studies, >9000 informal caregivers will be enrolled, representing family members, friends, or others who are a consistent part of the care of the patients participating. Caregiver outcome assessments include caregiver burden, health-related quality of life, satisfaction with patient care and the death experience, and measures of depression and anxiety, among others. These assessments will provide 
crucial insights into the needs of informal caregivers to the advanced illness population and inform how the health care team can better support these individuals alongside the patients they care for.

The interventions being compared in these trials vary by mode of delivery as well as by specialty of the clinicians delivering them. These include comparisons of in-person, telephone, and telehealth approaches to delivering care, as well as studies focused on the delivery of palliative care by board-certified palliative care physicians and other providers, including nurse practitioners, registered nurses, specialists (e.g., hepatologists), and primary care providers. These comparisons will improve understanding around the innovations in the health care delivery workforce that could promote transdisciplinary strategies to meet the needs of the growing population of older adults living with serious advanced illnesses. $^{7}$

\section{Stakeholder Engagement}

At time of application, research teams were required to demonstrate how patients and other national or regional health care system stakeholders would participate as partners in the proposed research throughout the study. ${ }^{16,19}$ The stakeholder partners that study teams have brought together within these awards are diverse and reflective of the key contributors to the palliative care landscape. These include faith leaders in communities, clinician and health system leaders, policy makers, eight unique payer organizations, and patients receiving palliative care and their caregivers. ${ }^{20} \mathrm{Al}-$ though patient and stakeholder engagement in PCORI's funded research awards is required and supported, study teams are encouraged to structure and operationalize engagement mechanisms to best suit their study design and vision for engagement. Across the nine palliative care studies, all use at least one advisory body as a means of collaboration and input, with some studies utilizing multiple advisory bodies, and one study utilizing as many as six different advisory bodies (divided by stakeholder type). In addition to the advisory bodies, three studies have patients or stakeholders as coinvestigators within their projects. The majority of the studies (five out of nine) have "decentralized" engagement structures, which means they have mechanisms to solicit input or collaboration at the awardee institution level as well as at the local site implementation level. Several of the studies have stakeholder organizations in common, with representatives from national palliative care advocacy and provider organizations serving as advisors on multiple projects. PCORI intends to utilize this built-in dissemination network as studies progress to assemble key stakeholders and gather input on how the findings from these studies could facilitate improvements in clinical practice.

\section{Promoting Success: Building a Network of Awardees}

Simultaneous funding of a collection of research studies with a similar focus presents a unique opportunity to create a cohort of awardees with the potential to expand the reach of this initiative beyond the individual studies themselves. PCORI has worked to build a learning network to both support the success of awardees in the conduct of these complex trials and to maximize their collective scientific impact through development of collaborative research products.
Early in the life cycle of these awards, PCORI operationalized this intention through organization of regular meetings of the awardees throughout the year, including an annual in-person meeting. Fostering these interactions among awardees allows for sharing of study progress to date, troubleshooting of common study challenges, and discussion of potential outputs as a collective group. Existing systematic reviews have observed a significant variation in the key outcome measures used across different palliative care studies, making it difficult to synthesize findings in a systematic manner. ${ }^{9,10}$ Consequently, to facilitate meaningful comparisons of findings across the nine trials, one early and extensive effort made as part of this learning network has been to ensure, to the extent possible, harmonization of outcome measures across multiple studies. As a result of this activity, several of the studies are now using common outcome measures for assessment of a variety of patient outcomes-for example, using the Patient-Reported Outcomes Measurement Information System (PROMIS ${ }^{\circledR}$ ) 10 and the Edmonton Symptom Assessment Scale to measure healthrelated quality of life and common symptoms experienced by patients, respectively. ${ }^{21,22}$

\section{Conclusion}

Given the aging of the population and an increasing demand for palliative care services, there is an urgent need to understand the most effective, efficient, and equitable ways of delivering palliative care in the community to patients living with serious advanced illnesses and their caregivers. PCORI, under direction from stakeholder input, has made a significant commitment and investment to address this unmet need by funding some of the largest multisite palliative care CER trials to date. The protocols of each of the nine PCORIfunded studies, detailed in this issue, demonstrate the expansive reach of the investment PCORI has made in an effort to further the research agenda and provide substantive research evidence in stakeholder-identified areas of need in the field of palliative care.

\section{Acknowledgments}

We would like to acknowledge the following PCORI staff for their assistance in data preparation and for facilitating the management of these studies: Sindhura Gummi, MPH; Subomi Akindoju, MPH; and Hamidat Segunmaru, MPH.

\section{Author Disclosure Statement}

The authors have no conflicts of interest to disclose.

\section{References}

1. Institute of Medicine: Dying in America: Improving Quality and Honoring Individual Preferences Near the End of Life. The National Academies Press, Washington, DC, 2015.

2. National Coalition for Hospice and Palliative Care: Clinical Practice Guidelines for Quality Palliative Care, 4e. 2018. https:/www.nationalcoalitionhpc.org/wp-content/uploads/ 2018/10/NCHPC-NCPGuidelines_4thED_web_FINAL.pdf (last accessed April 30, 2019).

3. Adelson K, Paris J, Horton JR, et al.: Standardized criteria for palliative care consultation on a solid tumor oncology 
service reduces downstream health care use. J Oncol Pract 2017;13:e431-e440.

4. Kavalieratos D, Corbelli J, Zhang D, et al.: Association between palliative care and patient and caregiver outcomes: A systematic review and meta-analysis. JAMA 2016;316: 2104-2114.

5. O'Connor NR, Moyer ME, Behta M, et al.: The impact of inpatient palliative care consultations on 30-day hospital readmissions. J Palliat Med 2015;18:956-961.

6. Temel JS, Greer JA, Muzikansky A, Gallagher ER, et al.: Early palliative care for patients with metastatic non-smallcell lung cancer. N Engl J Med 2010;363:733-742.

7. Center to Advance Palliative Care: 2015 State-by-State Report Card on Access to Palliative Care in Our Nation's Hospitals. 2015. https://reportcard.capc.org/wp-content/ uploads/2015/08/CAPC-Report-Card-2015.pdf (last accessed April 30, 2019).

8. Rowe JW, Fulmer T, Fried L: Preparing for better health and health care for an aging population. JAMA 2016;316: 1643-1644.

9. Lorenz KA, Lynn J, Dy SM, et al.: Evidence for improving palliative care at the end of life: A systematic review. Ann Intern Med 2008;148:147-159.

10. Dy SM, Aslakson R, Wilson RF, et al.: Closing the quality gap: Revisiting the state of the science (vol. 8: Improving health care and palliative care for advanced and serious illness). Evid Rep Technol Assess 2012;208: 1-249.

11. Houben CHM, Spruit MA, Groenen MTJ, et al.: Efficacy of advance care planning: A systematic review and metaanalysis. J Am Med Dir Assoc 2014;15:477-489.

12. National Institute of Nursing Research: 2013. Building momentum: The science of end-of-life and palliative care. A review of research trends and funding, 1997-2010. https://www.ninr.nih.gov/sites/files/docs/NINR-BuildingMomentum-508.pdf (last accessed May 30, 2019).

13. Patient-Centered Outcomes Research Institute: About Us. 2019. https://www.pcori.org/about-us (last accessed May 30, 2019).

14. Selby JV, Beal AC, Frank, L: The Patient-Centered Outcomes Research Institute (PCORI) national priorities for research and initial research agenda. JAMA 2012;307:1583-1584.

15. Arora NK, Gayer C, DiGioia K, et al.: A patient-centered approach to research on palliative care for patients with advanced illnesses and their caregivers. J Pain Symptom Management 2017;54:e1-e9.

16. Patient-Centered Outcomes Research Institute: PCORI Funding Announcement: Community-based palliative care delivery for adult patients with advanced illnesses and their caregivers. 2016. https://www.pcori.org/sites/default/files/ PCORI-PFA-2016-Cycle-3-Palliative-Care.pdf (last accessed May 30, 2019).

17. Patient-Centered Outcomes Research Institute: Patientcentered palliative care delivery for adults with advanced illnesses and their caregivers: Workgroup meeting summary. 2017. https://www.pcori.org/sites/default/files/PCORIPalliative-Care-Stakeholder-Workshop-Meeting-Summary030716.pdf (last accessed May 30, 2019).

18. Cohn J, Corrigan J, Lynn J, et al.: Community-Based Models of Care Delivery for People with Serious Illness. Discussion Paper. Washington, DC: National Academy of Medicine, 2017.

19. Patient-Centered Outcomes Research Institute: Engagement Rubric for Applicants. 2016. https://www.pcori.org/ sites/default/files/Engagement-Rubric.pdf (last accessed May 30, 2019).

20. Patient-Centered Outcomes Research Institute: PCORI's Stakeholders. 2018. https://www.pcori.org/about-us/ourprograms/engagement/public-and-patient-engagement/pcorisstakeholders (last accessed May 30, 2019).

21. Bruera E, Kuehn N, Miller MJ, et al.: The Edmonton Symptom Assessment System (ESAS): A simple method for the assessment of palliative care patients. J Palliat Care 1991;7:6-9.

22. Cella D, Yount S, Rothrock N, et al.: The Patient-Reported Outcomes Measurement Information System (PROMIS): Progress of an NIH Roadmap cooperative group during its first two years. Med Care 2007;45(5 Suppl 1):S3-S11.

Address correspondence to: Carly P. Khan, PhD, MPH, RN

Patient-Centered Outcomes Research Institute (PCORI) 1919 M Street, NW, 4th Floor Washington, DC 20036

E-mail: ckhan@pcori.org 